\title{
COQBLIN-SCHRIEFFER IMPURITY WITH LOCAL EXCHANGE COUPLING: A PERTURBATIVE APPROACH
}

\author{
P. STEFAŃSKI \\ Institute of Molecular Physics, Polish Academy of Sciences \\ Smoluchowskiego 17, 60-179 Poznań, Poland
}

\begin{abstract}
The Coqblin-Schrieffer one-impurity model with the additional local exchange interaction is studied within diagrammatic approach. The RudermanKittel-Kasuya-Yoshida-type local exchange interaction between $f$ electrons of the impurity and a channel of conduction electron $l=0$ partial waves is treated in the molecular field approximation. The perturbation expansion resummation for the Coqblin-Schrieffer hybridization mediated interaction vertex part is carried out in the ladder approximation yielding the formula for the Kondo temperature $T_{\mathrm{K}}$ decreasing with increased local exchange. Moreover, the temperature divergence of the susceptibility at $T_{\mathrm{K}}$ is shifted towards $T=0$. For some critical strength of the local exchange interaction the susceptibility approaches a Curie-type dependence as for an uncompensated impurity magnetic moment. A relation to the "Kondo disorder" model, which leads to the non-Fermi-liquid behavior, is discussed.
\end{abstract}

PACS numbers: 71.10.Hf, 71.20.Eh, 72.15.Qm, 75.20.Hr

Recently, non-Fermi-liquid (NFL) behavior, manifested in the temperature dependences of the specific heat $C / T \propto-\ln T$, magnetic susceptibility $\chi \propto$ $\left(1-T^{1 / 2}\right)$, and resistivity $\Delta \rho \propto T$, has been reported for some uranium- and cerium-based alloys. Nearly all NFL heavy-fermion materials are disordered alloys, and all are found in the neighborhood of a transition to magnetic order in the temperature-composition (pressure) diagram [1,2]. Several mechanisms have been proposed to explain NFL anomalies. These are (i) the multichannel Kondo effect, first introduced as two-channel quadrupolar Kondo effect by Cox [3], (ii) magnetic instability due to a quantum critical point at zero temperature [4-6], (iii) "Kondo disorder" model $[7,8]$ where the NFL behavior is associated by an inhomogeneous distribution of the Kondo temperatures due to the structural disorder. The aim of this work is to study the $T_{\mathrm{K}}$ dependence on the molecular field produced by the exchange interaction of $f$ electrons of the impurity with a non-hybridizing subband of conduction electrons. It is in correspondence with model (iii) where the Kondo temperature is allowed to change from one impurity site to another. 
We consider the Coqblin-Schrieffer model [9], which is believed to describe the low temperature Kondo anomalies observed in Ce compounds. Additionally, we introduce the non-hybridizing local exchange interaction of the Ruderman-KittelKasuya-Yoshida type (RKKY-type) between the conduction electron spin density and the localized magnetic moment of the impurity. Inclusion of this additional conduction electron channel leads to the non-Fermi-liquid behavior of the quasiparticles, as shown by renormalization group study $[10,11]$. Originally, the Coqblin-Schrieffer (C-S) Hamiltonian is written in the partial wave basis [9]. However, when the additional RKKY-type local exchange (LE) interaction is introduced to the Hamiltonian, it is more convenient to work with plane wave representation [9]. In this picture the $\mathrm{C}-\mathrm{S}$ exchange interaction corresponds to the spin-flip process present in the $s-d$ Kondo Hamiltonian [12]. In the partial waves representation the $H_{\mathrm{LE}}$ corresponds to the exchange interaction of the local magnetic moment with $l=0$ spin density conduction electron partial waves at the impurity site, whereas the hybridization involves $l=3$ partial waves. Thus, the Kondo and local exchange interactions involve two different conduction electron channels. The partial wave representation is more adequate when the renormalization of energy levels of the impurity is to be considered in detail. In this paper, we are mainly interested in the critical behavior caused by the $\mathrm{C}-\mathrm{S}$ interaction itself, rather than the level renormalization.

The two-band model Hamiltonian takes the form

$$
\begin{aligned}
H & =\sum_{k, \sigma} \varepsilon_{k} c_{k, \sigma}^{+} c_{k, \sigma}+\sum_{l, \sigma} \varepsilon_{l} c_{l, \sigma}^{+} c_{l, \sigma} \\
& -J_{\mathrm{hyb}} \sum_{k, k^{\prime}} \sum_{\sigma, \sigma^{\prime}} c_{k, \sigma}^{+} c_{k^{\prime}, \sigma^{\prime}} f_{\sigma^{\prime}}^{+} f_{\sigma}-J_{\mathrm{LE}} s \cdot \boldsymbol{S}_{f},
\end{aligned}
$$

where $\varepsilon_{k}\left(\varepsilon_{l}\right)$ is the energy of the hybridizing (non-hybridizing) subband of conduction electrons with the wave vector $k(l)$. The third term is $\mathrm{C}-\mathrm{S}$ mediated hybridization interaction; $c_{k, \sigma}$ and $f_{\sigma}$ are the annihilation operators of the conduction electron with momentum $k$ and spin $\sigma$ and of the electron with spin $\sigma$ in the localized orbital of the magnetic impurity. For simplicity, we neglect the orbital degeneracy in the magnetic impurity. In this form the C-S term is equivalent to the $s-d$ pseudospin Kondo Hamiltonian with $S=1 / 2$. The last term is the non-hybridizing spin-only $L E$ interaction.

Perturbation expansion with respect to the Coqblin-Schrieffer hybridization interaction has been summed up in the ladder approximation [13, 14]. The local exchange interaction is treated as a molecular field $H_{\text {mol }}$.

We introduce bare $f$-pseudofermion and s-electron propagators: $G_{f \sigma}\left(i \omega_{n}\right)=$ $\left(\mathrm{i} \omega_{n}-E_{\sigma}+H_{\mathrm{mol}}\right)^{-1}$ and $G\left(\mathrm{i} \omega_{n}, k\right)=\left(\mathrm{i} \omega_{n}-\varepsilon_{k}\right)^{-1} . E_{\sigma}$ is the impurity energy level with a quantum number $\sigma$. The second order integral equation for the vertex part can be obtained through application of the common field theoretic method for the Matsubara propagators [15]:

$$
\Gamma\left(\mathrm{i} \omega_{n}\right)=\left(-J_{\mathrm{hyb}}\right)^{2} \sum_{k^{\prime}} \sum_{\sigma, \sigma^{\prime}} \frac{1}{\beta} \sum_{\omega_{s}} G_{f \sigma}\left[\mathrm{i}\left(-\omega_{s}+\omega_{n}\right)\right] G\left(\mathrm{i} \omega_{s}, k^{\prime}\right) .
$$

The summation over $\omega_{s}$ has been performed with the use of the Poisson sum formula, and the summation over $k^{\prime}$ has been replaced by the integration over 
conduction electron energy band of the width $2 D$ and the constant density of states $\rho$. Integration of Eq. (2) by parts with respect to the conduction electron energy assuming sharp Fermi cut-off (this assumption is similar to the one made by Kondo [12]), and retaining the terms which are $H_{\text {mol }}$ dependent gives the equation for the second order vertex part of the form $\Gamma=f\left(H_{\mathrm{mol}}\right) \ln \left(D / k_{\mathrm{B}} T\right)$, where $f$ is the Fermi distribution function. Summation of the "parquet diagrams" [13] to the infinite order as a geometric series, gives the equation for $T_{\mathrm{K}}$, dependent on $H_{\mathrm{mol}}$, as a temperature for which the Coqblin-Schrieffer interaction diverges

$$
T_{\mathrm{K}}=D \exp \left[-(1 / 2) J_{\text {hyb }} \rho f\left(H_{\mathrm{mol}}\right)\right] \text {. }
$$

Thus, we have obtained a self-consistent equation for $T_{\mathrm{K}} ; T_{\mathrm{K}}$ appears also in the denominator of the Fermi function argument on the right hand side of the equation. The graphical solution of Eq. (3) is shown in the insert of Fig. 1 for parameters listed in the caption. $T_{\mathrm{K}}$ decreases from $T_{\mathrm{K}}=12.73 \mathrm{~K}$ to $0 \mathrm{~K}$ when the interaction with the non-hybridizing conduction electron channel increases.

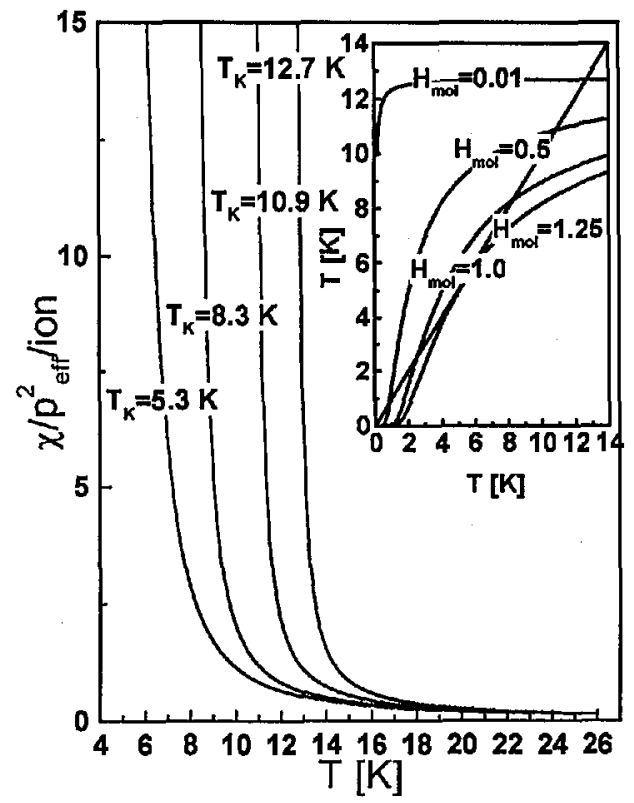

Fig. 1. Temperature dependence of the susceptibility for various values of $H_{\mathrm{mol}}$ given in kelvins; the graphical solution of Eq. (3) is shown in the insert. The curves were calculated for $D=10^{4} \mathrm{~K}$ and $J_{\text {hyb }} \rho=0.15$ [9].

The logarithmic divergence of the hybridization interaction and the dependence of the Kondo temperature on $H_{\text {mol }}$ has its influence on the static susceptibility $\chi . \chi$ divergence in $T_{\mathbf{K}}$, obtained from summation of the most divergent terms [14], also depends on $H_{\text {mol }}$ now. Thus, we obtain an equation for the susceptibility

$$
\chi(T)=\frac{\left(g \mu_{\mathrm{B}}\right)^{2} S(S+1)}{3 k_{\mathrm{B}} T}\left[1+\frac{2 J_{\mathrm{hyb}} \rho}{1-2 J_{\mathrm{hyb}} \rho f\left(H_{\mathrm{mol}}\right) \ln \left(D / k_{\mathrm{B}} T\right)}\right] .
$$


The shift of the divergence of the susceptibility at $T_{\mathrm{K}}$ is displayed in Fig. 1. For some value of $H_{\text {mol }}$ there is only a solution of Eq. (3) for $T_{\mathrm{K}}$ equal to $0 \mathrm{~K}$ and the temperature dependence of the susceptibility is of the Curie-type, as for uncompensated magnetic moment of the impurity.

It is worth emphasizing that the calculated susceptibility is obtained for one impurity and not for the sample. If the value of the LE interaction varies from one site of the impurity to another, then the distribution of the Kondo temperatures appears. The slight differences in $H_{\mathrm{mol}}$ are enhanced by the exponent appearing in Eq. (3), and can produce a distribution of the Kondo temperatures. The divergence of the susceptibility is smeared out by this distribution and instead of the plateau of $\chi$ in the low temperature region the $\chi \propto\left(1-T^{1 / 2}\right)$ dependence can appear, indicating NFL behavior.

\section{References}

[1] P. Gegenwart, F. Kromer, M. Lang, G. Sparn, C. Geibel, F. Steglich, Phys. Rev. Lett. 82, 1293 (1999).

[2] M.C. De Andrade, R. Chau, R.P. Dickey, N.R. Dilley, E.J. Freeman, D.A. Gajewski, M.B. Maple, R. Movshovich, A.H.C. Neto, G. Castilla, B.A. Jones, Phys. Rev. Lett. 81, 5620 (1998).

[3] D.L. Cox, Phys. Rev. Lett. 59, 1240 (1987).

[4] M.A. Continentino, Phys. Rev. B 47, 11587 (1993).

[5] A.J. Millis, Phys. Rev. B 48, 9887 (1993).

[6] H. v. Löhneysen, T. Pietrus, G. Portisch, H.G. Schlager, A. Schröder, M. Sieck, T. Trappmann, Phys. Rev. Lett. 72, 3262 (1994).

[7] V. Dobrosavljević, T.R. Kirkpatrick, G. Kotliar, Phys. Rev. Lett. 69, 1113 (1992).

[8] O.O. Bernal, D.E. MacLaughlin, A. Amato, R. Feyerherm, F.N. Gygax, A. Schenck, R.H. Heffner, L.P. Le, G.J. Nieuwenhuys, B. Andraka, H. v. Löhneysen, O. Stockert, H.R. Ott, Phys. Rev. B 54, 13000 (1996).

[9] B. Coqblin, J.R. Schrieffer, Phys. Rev. 185, 847 (1969).

[10] E. Pavarini, L.C. Andreani, Phys. Rev. Lett. 77, 2762 (1996).

[11] E. Pavarini, L.C. Andreani, Physica B 230-232, 523 (1997).

[12] J. Kondo, Solid State Phys. 23, 183 (1969).

[13] A.A. Abrikosov, Physics 2, 5 (1965).

[14] A.C. Hewson, The Kondo Problem to Heavy Fermions, Cambridge University Press, Cambridge 1993, p. 49.

[15] A.A. Abrikosov, L.P. Gorkov, I.E. Dzyaloshinski, Methods of Quantum Field Theory in Statistical Physics, Dover Publ. Inc., New York 1975, p. 97. 